\title{
Creciente importancia de las Revisiones Sistemáticas Cochrane en Evidencia
}

Ciapponi A. The Rising Importance of Cochrane Systematic Reviews in a Latin-American Evidence-Base Journal. XIII Cochrane Collaboration Colloquium. 22 al 26 de Octubre de 2005. Melbourne, Australia

\section{Introducción}

La "literatura secundaria", aquella que valora críticamente los estudios originales, está aumentando sostenidamente. Las revistas basadas en la evidencia (RBE) son publicaciones revisadas por pares, como ACP Journal Club, Evidence-Based Medicine, Evidence-Based Nursing, and Evidence-Based Mental Health, que identifican y evalúan artículos recientes de las revistas médicas más prominentes a través del riguroso criterios metodológicos. "EVIDENCIA" es una RBE bimensual producida en Argentina desde 1998 y como las otras, contiene resúmenes y comentarios de estudios originales y revisiones sistemáticas de buena calidad metodológica.

\section{Objetivos}

Determinar la proporción de las revistas más selectas y de las revisiones sistemáticas (Cochrane y no Cochrane) resumidas a lo largo de tiempo en "EVIDENCIA" en el contexto con otras RBE. Los objetivos:

\section{Métodos}

Se evaluaron los años 1998, 2000, 2002 y 2004. La proporción de RBE se obtuvo del artículo de Walker and Haynes1. Las RBE seleccionadas fueron: N England Journal of Medicine, JAMA, BMJ, Lancet y Cochrane Database Systematic Reviews.

\section{Resultados}

La proporción de revisiones sistemáticas resumidas ha aumentado desde que "EVIDENCIA" empezó a publicarse en 1998, cuando representaban el 17\% de los resúmenes (RBE 30\%). Para 2004 la proporción de revisiones sistemáticas era $28 \%$ (RBE 38\%). Las revisiones Cochrane tuvieron un crecimiento aún más marcado. De todas las revisiones, las Cochranes fueron 0\% (RBE 24\%) en 1998 y $43 \%$ (RBE $31 \%$ ) en 2004. El total de RBE selectas representó el $70 \%$ (RBE 38\%) en 1998 y 64\% (RBE 55\%) en 2004. Ver tabla 1.

\section{Conclusiones}

Las revisiones sistemáticas, especialmente Cochrane, han crecido, constituyéndose en una fracción sustancial de la literatura secundaria tanto en "Evidencia" como en las otras RBE.

Tabla 1 Resúmenes contenidos en la Revistas basadas en la evidencia (RBE) y EVIDENCIA (E) en 1998, 2000, 2002 y 2004.

\begin{tabular}{|c|c|c|c|c|c|c|c|c|}
\hline \multirow{3}{*}{ Revistas } & \multicolumn{8}{|c|}{ Proporción respecto al total de contenidos (\%) } \\
\hline & \multicolumn{2}{|c|}{1998} & \multicolumn{2}{|c|}{2000} & \multicolumn{2}{|c|}{2002} & \multicolumn{2}{|c|}{2004} \\
\hline & RBE & $\bar{E}$ & $\overline{\mathrm{RBE}}$ & $\bar{E}$ & RBE & $\mathbf{E}$ & RBE & $\bar{E}$ \\
\hline NEJM & 9,9 & 20,2 & 7,5 & 31,5 & 9,2 & 20,6 & 10,7 & 18,0 \\
\hline JAMA & 9,2 & 21,4 & 9,9 & 28,8 & 6,8 & 23,8 & 14,8 & 20,0 \\
\hline BMJ & 8,0 & 10,7 & 6,0 & 4,1 & 7,5 & 12,7 & 8,3 & 10,0 \\
\hline Lancet & 4,8 & 17,9 & 10,4 & 9,6 & 7,0 & 12,7 & 8,0 & 4,0 \\
\hline Cochrane & 7,0 & 0,0 & 7,2 & 0,0 & 15,7 & 9,5 & 13,5 & 12,0 \\
\hline Otras & 61,1 & 29,8 & 59,0 & 26,0 & 53,8 & 20,7 & 44,7 & 36,0 \\
\hline Total & 38,9 & 70,2 & 41,0 & 74,0 & 46,2 & 79,3 & 55,3 & 64,0 \\
\hline
\end{tabular}

Agustín Ciapponi [ Unidad de Medicina Familiar y Preventiva. Hospital Italiano de Buenos Aires. ]

Referencias

1. Walker C, Haynes B. The growing prominence of Cochrane systematic reviews in evidence-based resources.

http://www.cochrane.org/colloquia/abstracts/ottawa/O-021.htm

\section{INFO - EVIDENCIA}

\section{¿Qué es Evidencia, Actualización en la Práctica Ambulatoria?}

Una publicación independiente editada cada dos meses desde 1997 por la Fundación MF, Organización sin fines de lucro dedicada promover el desarrollo de la medicina familiar y la atención primaria de la salud.

\section{¿Cuál es el objetivo de Evidencia?}

Contribuir a la educación continua y a la actualización de los profesionales de la salud de la región en el área de la atención ambulatoria.

\section{¿Contenidos de Evidencia}

- Información independiente, actualizada y resumida en forma sencilla y en castellano sobre temas relevantes de la atención ambulatoria.

- Artículos seleccionados por su calidad y relevancia clínica, resumidos y comentados críticamente por destacados profesionales del área.

- Revisiones sobre temas clínicos, epidemiológicos, sanitarios o humanísticos, de importancia para la práctica ambulatoria.

Notas farmacológicas para la actualización rápida de temas de terapéutica clínica.

Discusión de casos clínicos con herramientas de Medicina Basada en la Evidencia.

Glosario de términos de epidemiología y medicina basada en la evidencia

¿Cómo es posible acceder a Evidencia?

Por suscripción a la edición en papel, o bien por Intenet, en nuestro sitio:

http://www.evidencia.org 